\title{
First observation of 3D aggregates in a single-component Langmuir film below the equilibrium spreading pressure
}

\author{
George R. Ivanov ${ }^{1,2}$ and Zorica Polevska ${ }^{2}$ \\ ${ }^{1}$ NanoBioSense Ltd., compl. Yavorov, bl. 38, entr. B, 1111 Sofia, Bulgaria \\ ${ }^{2}$ University Laboratory for Nanoscience and Nanotechnology, University of Architecture, Civil Engineering and Geodesy, \\ blvd. Hr. Smirnenski 1, 1046 Sofia, Bulgaria
}

\begin{abstract}
Single component monolayers from Dipalamitoyl Phosphatidyl Ethanolamine head labelled with the fluorescent chromophore NitroBenzoxaDiazole (DP-NBD-PE) were investigated at the air-water interface as Langmuir films and deposited on silicon wafers or glass plates as Langmuir-Blodgett (LB) films. A step compression and monitoring of the pressure relaxation together with domain formation as observed with Brewster angle microscopy suggests that main transition from liquid expanded to liquid compressed state start at around $6.9 \mathrm{mN} / \mathrm{m}$ though on the isotherm it starts around $9 \mathrm{mN} / \mathrm{m}$ at $25^{\circ} \mathrm{C}$. Brewster angle microscopy also reveals a nonuniform structure in the monolayer. 3D aggregates - cylinders with $50-150 \mathrm{~nm}$ diameter and bilayer height were observed with Atomic Force Microscopy when deposition was carried at $7 \mathrm{mN} / \mathrm{m}$, above the main phase transition but considerably lower than the equilibrium spreading pressure of $19.6 \mathrm{mN} / \mathrm{m}$ for this molecule. When LB film deposition is carried just below the main phase transition a uniform height layer of film in a liquid phase is observed with AFM with no structures. Both compression and deposition were carried at very low speeds with large time to relax in ordered to avoid kinetic effects. These 3D aggregates are not due to the transfer process or interaction with the substrate. These aggregates provide a highly developed area combined with monolayer thick structure which can produce very fast and highly sensitive biosensors.
\end{abstract}

\section{Introduction}

Langmuir-Blodgett film field has matured in the past 30 years. However systematic investigation of a structurally complex molecules still allows discovery of new effects. We have started the investigation of fluorescently labelled phospholipids some 28 years ago and over the years we have discovered 3 new effects [1,2]. We have focused our efforts on fluorescently head labelled phospholipid Dipalmitoyl-Nitrobenzoxadiazole-Phosphatidyl

Ethanolamine (DP-NBD-PE). Our focus in such type of compound was due to 2 reasons: the biomimetic aspect of a research of a molecule which is similar to the lipids in biological membranes and understanding of these more complex molecules. NBD chromophore is considered most suitable for optical biosensors due to high sensitivity to the environment in close proximity [3].

Here we elaborate on recently discovered new effect formation of stable 3D nanosized aggregates at pressures considerably lower than the equilibrium spreading pressure (ESP). In previous research there was a considerable debate on $3 \mathrm{D}$ aggregates. But these structures were observed above the ESP in which the monolayer is in a metastable state. Or it was a mixture of several compounds. Instantaneous nucleation was proposed as a mechanism of collapse [4] but at high, collapse surface pressures. Sequential collapse in Langmuir films was also observed starting in the solid phase region [5] but no information on the ESP was provided in this case. In most cases, the $\pi-\mathrm{A}$ isotherms of amphiphiles are in a metastable state above the ESP and are in a state of supersaturation. This region of supersaturation is confined by ESP and the "collapse pressure" at which the irreversible monolayer collapse starts. The main characteristics of the supersaturated state is that the monolayers held at constant area per molecule or constant surface pressure show relaxation phenomena discussed in the terms of slow collapse.

\section{Materials and methods}

1,2-dipalmitoyl-sn-glycero-3-phosphoethanolamine-N(7-nitro-2-1,3-benzoxadiazol-4-yl) (DP-NBD-PE) (figure 1) in chlorophorm solution was purchased from Avanti Polar Lipids. The claimed purity was above $99 \%$ and was used without further purification. This is a head labelled phospholipid with two saturated 16 carbon atoms tales. For

\footnotetext{
* Corresponding author: george $@$ at-equipment.com
} 
the Langmuir film data presented here a newly ordered and just opened compound was used.

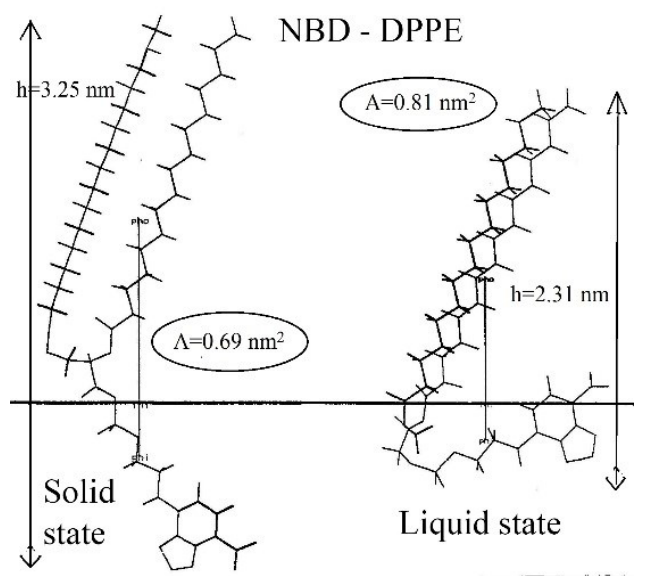

Fig.1 Structure of the DP-NBD-PE molecule and its most probable conformations according to molecular conformational analysis at simulated air-water interface in solid and liquid state.

$\pi-\mathrm{A}$ isotherms were measured on a long trough from KSV-NIMA (Finland). Together with the isotherms surface potential and Brewster angle microscopy (BAM) were measured, both manufactured from the same company. For the experiment shown a compression in steps of 2-4 $\mathrm{mN} / \mathrm{m}$ was performed and then the equilibration of the system was monitored over time. For the AFM data a very slow constant velocity compression was used prior to the LB deposition which was also carried at extremely low withdrawal velocity.

For the Atomic Force Microscopy (AFM) an LB monolayer film was deposited on the upstroke on Si wafer substrate at different surface pressures. Si wafer was used because it has less surface roughness. Anfatec (Germany) Level AFM was used in dynamic mode.

\section{Results and discussion}

Surface pressure - mean molecular area $(\pi-A)$ isotherms at different temperatures on pure water or in the presence of different metal counter ions was presented earlier [6]. Measurements were combined with fluorescence microscopy of the Langmuir film. The main phase transition from liquid expanded to liquid condensed phase at $20^{\circ} \mathrm{C}$ on pure water was at $6.9 \mathrm{mN} / \mathrm{m}$. On figure 2 a more precise measurement of the isotherm is shown with compression in steps. After a step the film was maintained at constant surface pressure and monolayer relaxation was monitored through barrier position movement over time. Surface potential was measured simultaneously and BAM images were taken. They are shown in figures 2, 3 and 4 at increasing surface pressures.

At first look the main phase transition starts at $25{ }^{\circ} \mathrm{C}$ at surface pressure around $9 \mathrm{mN} / \mathrm{m}$ with a typical almost horizontal plateau. But looking at the barrier position vs time data (not shown) one can see that the phase transition starts below $6 \mathrm{mN} / \mathrm{m}$. When we maintained constant surface pressure at $6 \mathrm{mN} / \mathrm{m}$ which is evidenced with the horizontal region on figure 2 the average barrier speed increases with time indicating that some molecules transfer from liquid to solid conformation. Formation of solid domains at this pressure is observed with BAM (figure 3).

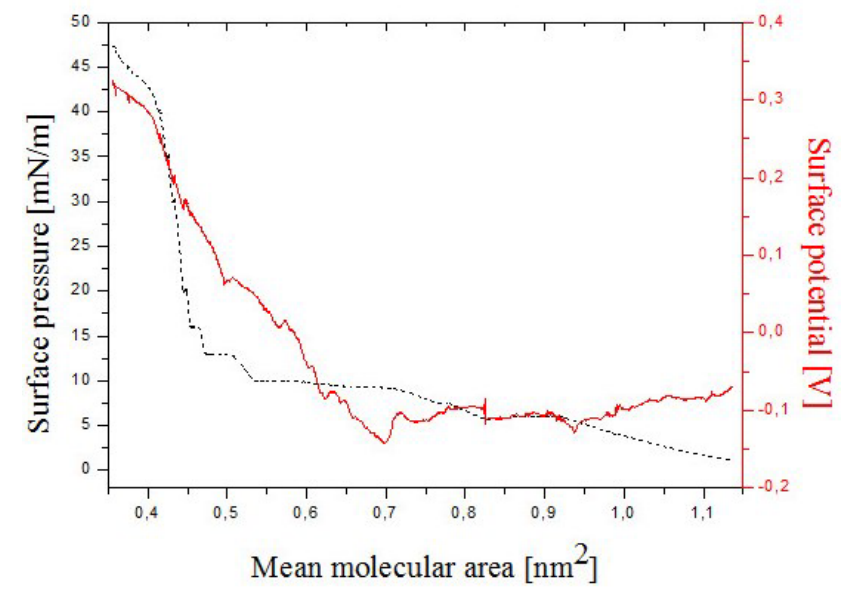

Fig. 2. Surface pressure (dotted line) and Surface potential as function of mean molecular area for a Langmuir film from DPNBD-PE on pure water at $25^{\circ} \mathrm{C}$.

In the middle of the main phase transition at mean molecular areas (MMA) around $0.65 \mathrm{~nm}^{2}$ the surface potential starts to increase (figure 2). At $20^{\circ} \mathrm{C}$ the extrapolated to zero surface pressure of solid phase was $0.66 \mathrm{~nm}^{2}$ which is in excellent agreement with our molecular conformational analysis at simulated air-water interface which predicts a MMA of the solid phase of 0.69 $\mathrm{nm}^{2}$ [6]. The main absorption band at $460 \mathrm{~nm}$ for NBDlabelled lipids is due to intramolecular charge transfer [7], which is accompanied by a large ( 4 Debye) change in dipole moment [8]. As the experiments here were carried in light this dipole moment is present. Molecular conformational analysis at simulated air-water interface suggests that in liquid phase the head group region is horizontal, even pointing a bit up towards the air-water interface while in the solid phase it point almost vertically down towards water bulk (see figure 1). There is almost $90^{\circ}$ change in the dipole moment orientation which gives excellent contrast when scanning surface potential microscopy was used [9]. This middle region of the plato of the main phase transition corresponds to figure 4 . Circular domains from the liquid compressed phase have formed. The domains are with different diameters, suggesting a second generation of crystallization centres. On a higher resolution inspection with fluorescence microscopy [10] and AFM [6] the domains are initially almost circular but at higher pressure become dendridic. This shape change is due to a balance between the line surface tension which tries to keep domains circular minimizing their perimeter and the repulsive forces between similarly oriented molecular dipoles which tries to separate molecules further apart. On a higher resolution AFM of a DP-NBD-PE LB films the solid domains do not have a strict boundary line but are composed of "archipelagos" and small islands floating in the liquid phase "sea".

At higher surface pressures the solid domains get in touch with each other (figure 5) and the slope of the $\pi-\mathrm{A}$ isotherm increases. The surface potential continues to grow almost linearly due to a larger number of solid domains entering the area below the vibrating electrode. 


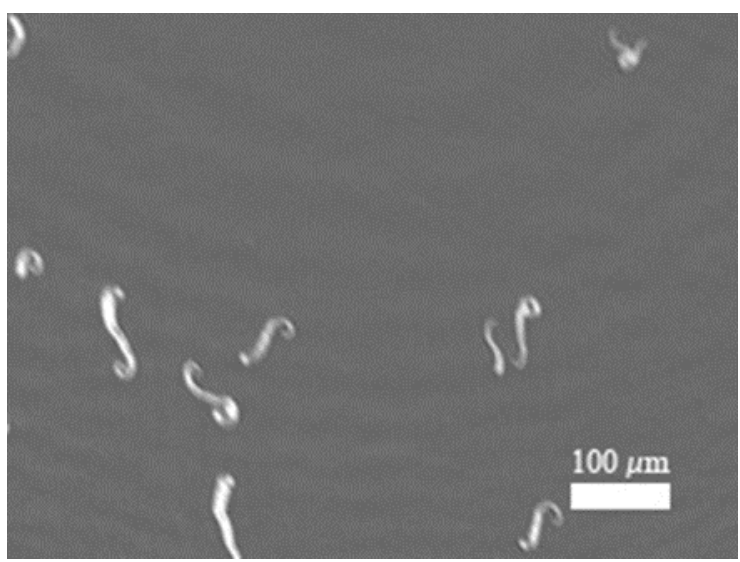

Fig. 3. BAM from a DP-NBD-PE Langmuir film at surface pressure $5.9 \mathrm{mN} / \mathrm{m}$ and mean molecular area of $0.82 \mathrm{~nm}^{2}$.

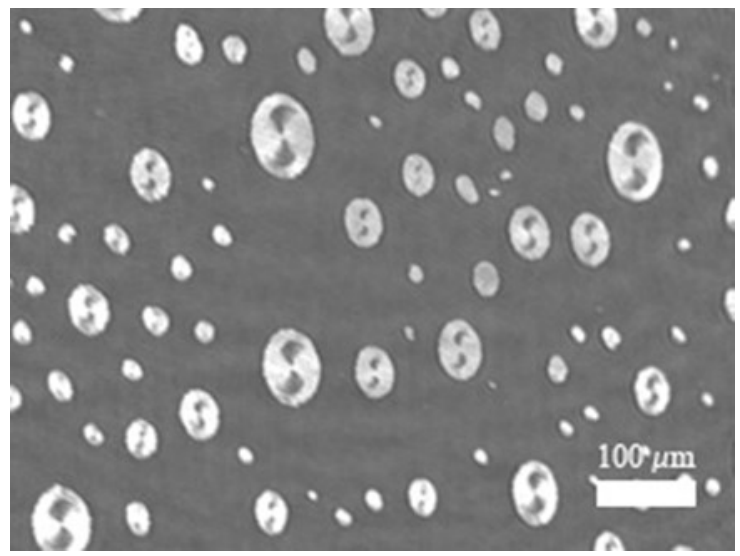

Fig. 4. BAM from a DP-NBD-PE Langmuir film at surface pressure $9.35 \mathrm{mN} / \mathrm{m}$ and mean molecular area of $0.65 \mathrm{~nm}^{2}$.

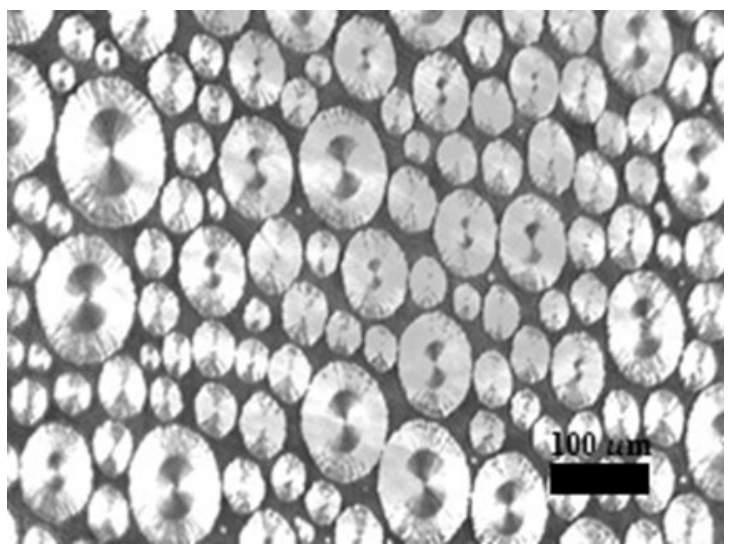

Fig. 5. BAM from a DP-NBD-PE Langmuir film at surface pressure $13 \mathrm{mN} / \mathrm{m}$ and mean molecular area of $0.47 \mathrm{~nm}^{2}$.

On figure 5 the structure of the solid domain is clearly seen. It is composed of a central circular region with 2 pie type sections with different molecular tilt orientations. Around this central region is another region composed of smaller stripes with not so high polarization contrast. Initially domains are circular growing from a single nucleation centre. Afterwards at increasing surface pressure smaller domains start to attach to the bigger central domain [10]. This mechanism was observed also in a Langmuir film from DP-NBD-PE at $5^{\circ} \mathrm{C}[6]$ where higher surface pressure was needed to overcome the repulsion between the domains and they start to merge.

On figure 6 the formation of 3D nanosized aggregates in a single component LB film deposited significantly below the ESP which for this molecule was measured to be $19.6 \mathrm{mN} / \mathrm{m}$ [6] is shown. When LB film deposition is carried below the main phase transition a uniform liquid phase is observed with AFM. However just above the beginning of the main phase transition at around $7 \mathrm{mN} / \mathrm{m}$ cylindrical structures with bilayer height are observed. If the deposition is carried out at higher than the ESP surface pressures these cylinders grow in height initially up to 13 $\mathrm{nm}$ for deposition at $33 \mathrm{mN} / \mathrm{m}$ and up to $35 \mathrm{~nm}$, and in some cases to hundred nanometers at $43 \mathrm{mN} / \mathrm{m}$. However, if the monolayer is allowed to relax under normal laboratory conditions for some time ( 50 days in this case), those cylinders again become with bilayer height [6]. Typical diameter of the cylinders is 50 to $150 \mathrm{~nm}$.

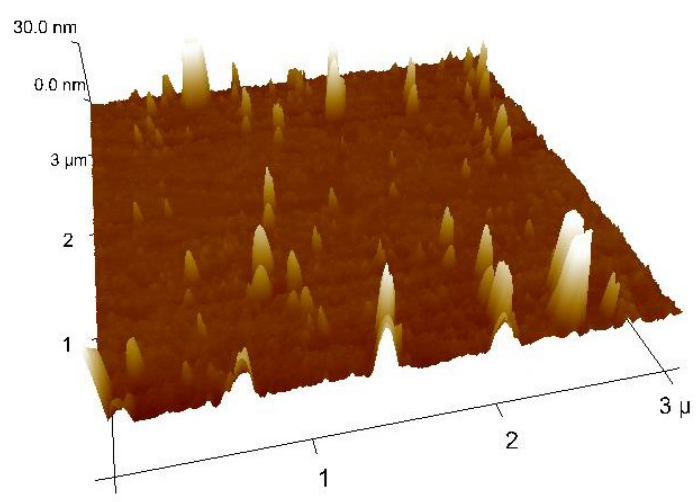

Fig. 6. AFM of an LB film monolayer from DP-NBD-PE transferred at $10 \mathrm{mN} / \mathrm{m}$, room temperature and pure water.

These cylinders can be due to several artefacts. Contamination can be excluded because the substance is more than $99 \%$ pure and all other typical precautions were taken. Kinetic effects can also be eliminated because there is a complete match between the fluorescence microscopy pictures of the Langmuir film and the LB monolayer on the substrate [11]. The biggest artefact problem can be whether there are no alterations during the transfer process from the air-water interface to the solid substrate, i.e. whether these $3 \mathrm{D}$ cylinders are not a result of the LB film deposition or an interaction with the substrate. It seems that this depends on the substance transferred. H. Riegler used a tilted substrate and a fluorescence microscope to monitor the lipid film deposition with $2 \%$ label [12]. With the substrate stationary, upon increasing the surface pressure, substrate mediated condensation of probedepleted stripes was observed. During the slow LangmuirBlodgett transfer (i.e. upstroke of the substrate under constant surface pressure), a domain-free gap is observed adjacent to the three-phase line. Both phenomena were caused by the substrate mediated condensation of the monolayer onto the solid surface in the moment of its deposition. On the other hand D. Vollhardt et al. investigating 1-monostearoylglycerol monolayers and comparing BAM and friction mode AFM data suggests that after the deposition of the monolayer, the main features of the condensed phase morphology, such as size, 
shape, and azimuthal tilt, are preserved independent of the solid substrate [13]. Similar shape and size of the condensed phase domains for two different systems were also found [14]. So it seems highly unlikely that these 3D aggregates in our experiment are formed during the deposition process. Further on they were observed on quite large number of experiments and depositions both on $\mathrm{Si}$ wafer or glass substrates.

We have not measured directly the structure of these 3D cylinders but some integral measurements can help. Data from our Stark spectroscopy measurements show that DP-NBD-PE molecules tend to form centrosymmetrical non-polar structures. Thus for the second layer in the bilayer structures the molecules most probably flip over and we have a tail - tail contact. Comparing fluorescence lifetime imaging microscopy data with integrally measured fluorescence lifetime we can suggest that the molecules in the 3D cylinders are in the liquid phase with much higher fluorescence lifetime compared to the solid phase [15].

\section{Conclusion}

The combination of $\pi-A$ isotherm with step compression and monitoring of the relaxation processes, surface potential, BAM and AFM measurements gives detailed picture of the behaviour of DP-NBD-PE single component monolayers both as Langmuir and LB films. Current measurements are combined with our systematic previous research on this compound using almost all possible experimental methods and computer-aided molecular conformational analysis at simulated air-water interface. When the LB film deposition was carried above $7 \mathrm{mN} / \mathrm{m}$ at $20^{\circ} \mathrm{C}$ where the liquid expanded to liquid condensed phase transition begins, 3D aggregates with bilayer height and 50 to $150 \mathrm{~nm}$ diameters are observed. This deposition pressure is significantly below the ESP suggesting that these are not some metastable effects. These cylinders are stable over time at least on a months scale.

\section{Acknowledgments}

This work is part of NanoBioSense Ltd. development program. It was also supported by a grant number 176/15 with the Centre for Design and Innovation of the University of Architecture, Civil Engineering and Geodesy, Sofia, Bulgaria. Invitation to visit and perform some measurements in the laboratory of KSV-NIMA from Biolin Scientific group is gratefully acknowledged. Special thanks to Jyrki Korpela from KSV-NIMA for the help with the measurements.

\section{References}

1. G.R. Ivanov, "First observation of fluorescence selfquenching in Langmuir films", Chem. Phys. Lett., 193323 (1992)

2. G.R. Ivanov and M. Fujihira, "Novel phenomena in organized organic monolayers", in Thin Film Materials and Devices - Developments in Science and Technology, eds. J. M. Marshall, N. Kirov, A. Vavrek, J. M. Maud, World Scientific, Singapore, 261 (1999)

3. J.D. Brennan, K.M.R. Kallury and U.J. Krull, Thin Solid Films, 244, 898 (1994)

4. D. Vollhardt, Colloids and Surfaces A, 156, 79 (1999)

5. E. Hatta, Langmuir, 20, 4059 (2004)

6. G.R. Ivanov, G. Georgiev and Z. Lalchev, Fluorescently Labeled Phospholipids - New Class of Materials for Chemical Sensors for Environmental Monitoring, in Relevant Perspectives in Global Environmental Change, ed. J. Agboola, ISBN: 978953-307-709-3, Rijeka: (InTech) 89 (2011)

7. P.A. Paprica, N.C. Baird and N.O. Petersen. J. Photochem. Photobiol. A: Chem., 70, 51 (1993)

8. S.A. Mukherjee, A. Chattopadhyay, A. Samanta and T. Soujanya, Phys. Chem., 98, 2809 (1994)

9. G.R. Ivanov and M. Fujihira, "Single component Langmuir-Blodgett film investigated with 5 scanning probe microscopy methods", Colloids and Surfaces A, 198 - 200, 305 (2002)

10. G.R. Ivanov and N.I. Geshev, „New effects in Langmuir and Langmuir-Blodgett monolayers from fluorescently labelled phospholipids and their possible use for water quality control", IOP Science, Journal of Physics: Conference Series, 682, 012037 (2016)

11. G.R. Ivanov, J. Nanoscience and Nanoengineering, 2, 15 (2016).

12. H. Riegler and K. Spratte, Thin Solid Films, 210 - 211, 9 (1992)

13. K. Meine, D. Vollhardt and G. Weidemann, Langmuir, 14, 1815 (1998).

14. L. Santesson, T.M.H. Wong, M. Taborelli, P. Descouts, M. Liley, C. Duschl, H. Vogel, J. Phys. Chem. 99, 1038 (1995).

15. G.R. Ivanov and J. Burov, AIP Conf. Proc. 899, 257 (2007) 